EUROTeV-Report-2007-034

\title{
BEAM PROFILE MEASUREMENTS WITH THE 2-D LASER-WIRE AT PETRA*
}

\author{
M. T. Price, G. A. Blair, G. Boorman, S. T. Boogert, A. Bosco", S. Malton, \\ John Adams Institute at Royal Holloway University of London, Egham, UK \\ T. Kamps, BESSY GmbH, Berlin, Germany
}

K. Balewski, E. Elsen, V. Gharibyan, H-C. Lewin, F. Poirier, S. Schreiber, N. Walker, K. Wittenburg DESY, Hamburg, Germany.

\section{Abstract}

The current PETRA II Laser-Wire system, being developed for the ILC and PETRA III, uses a piezodriven mirror to scan laser light across an electron bunch. This paper reports on the recently installed electron-beam finding system, presenting recent horizontal and vertical profile scans with corresponding studies.

\section{INTRODUCTION}

Laser based beam profile monitors and in particular the laser-wire (LW) have proven to be the only way to measure electron (or positron) bunch profiles when several factors might limit the usability of traditional profiling methods such as wire scanners or screens. For example, when spot-sizes to be measured go below the micron scale [1], or the bunch current or energy become too high and a physical wire will be destroyed. Furthermore, LWs are also essentially non-invasive.

The principle on which the LW is based is the inverse Compton scattering produced in the collisions between electrons and laser photons. By counting the number of Compton scattered photons produced as a function of the laser position, it is possible to reconstruct the spatial electron distribution. Several aspects (and limits) of the LW are being considered, such as the achievable resolution [1], speed [2] [3], but most important, its reliability as an electron beam diagnostics tool.

We describe here the upgrade of the $1 \mathrm{D}$ (vertical) LW tested previously at PETRA [4] to a 2D bunch profiler, where the laser can be sent to collision in either the horizontal or the vertical direction alternatively. In this paper we describe the major upgrades and differences with the previous $1 \mathrm{D}$ scanner.

\section{2-D SCANNER FEATURES}

The experimental setup of the $2 \mathrm{D} \mathrm{LW}$ is shown in Fig.1. The high power laser is installed in a laser-lab connected through a pipe with the accelerator tunnel. The laser beam is transported from the source to the vertical breadboard by means of a two-lens relay system which conjugates the images of the laser beam in two different positions: $3 \mathrm{~m}$ before the first lens and $6 \mathrm{~m}$ after the second.

Once in the tunnel, the beam is first expanded to approximately $30 \mathrm{~mm}$ diameter and collimated by a telescope (in order to obtain the tightest spot-size at the

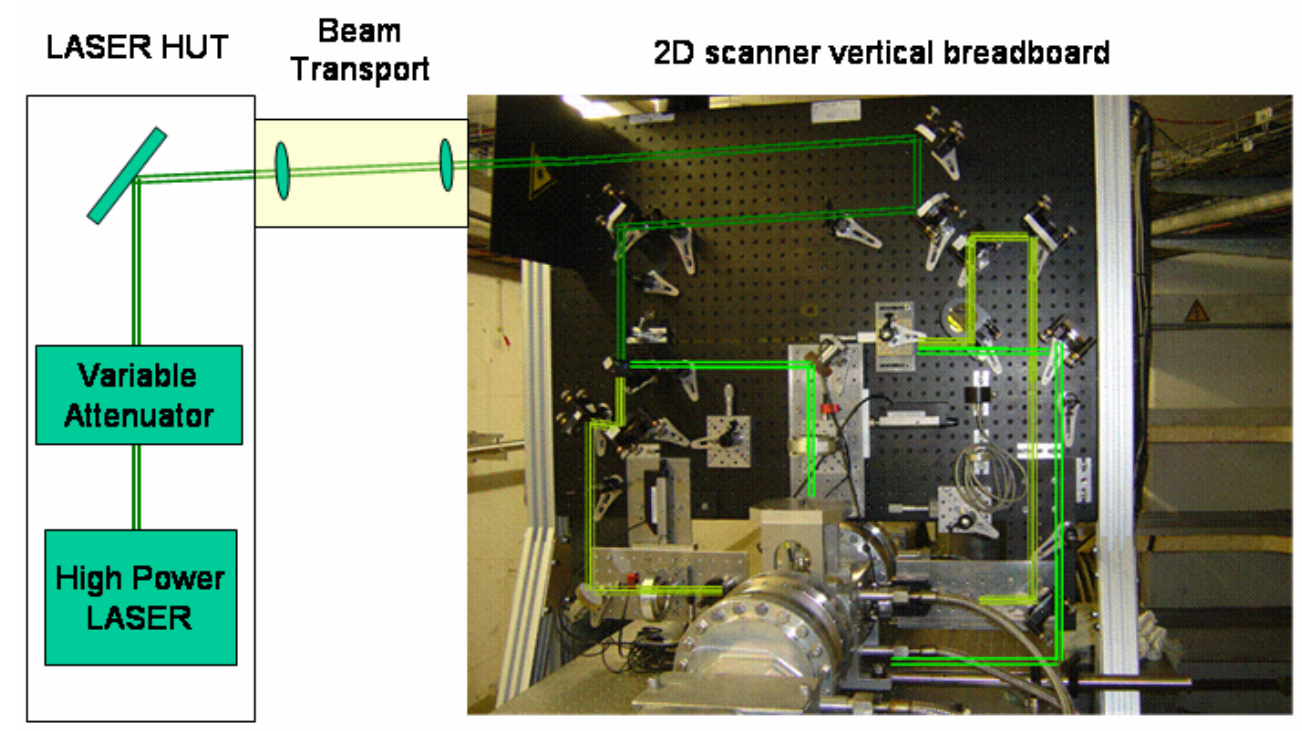

Figure 1. Representation of the LW experimental setup. The laser light is transported from the lab to the vertical breadboard using a relay imaging system. The two different paths (VP and HP) are traced using different colours.

\footnotetext{
*Work supported by the PPARC LC-ABD collaboration and the Commission of the European Communities under the 6th Framework Programme Structuring the European Research Area, contract nr RIDS-RIDS-011899. EUROTeV nr: EUROTeV-Report-2007-034

\# alessio.bosco@rhul.ac.uk
} 
interaction plane) and then sent to a motorized flipper mirror which acts as a remotely controllable path selector. Depending on the flipper mirror position, the laser beam is driven to a different scanning arm, vertical profiler (VP) or horizontal profiler (HP), towards the interaction point (IP) in the beam-pipe.

\section{Pre-IP}

The core of the LW scanner (either the VP or HP) is sketched in Fig. 2. It consists of a piezo-driven mirror (with total scanning range of approximately $2 \mathrm{mrad}$ ) and a focusing lens ( $\mathrm{f}=250 \mathrm{~mm}$, air spaced doublet) mounted on a rigid plate which can be moved transversally by a remotely controllable motorized translation stage.

This enables us to move the laser beam to find collisions with the positron bunch. Furthermore, the lens itself is mounted on an independent longitudinally movable motorized translation stage which allows positioning the laser beam waist. This is essential for setting the laser beam focus position to be at the centre of the electron beam distribution.

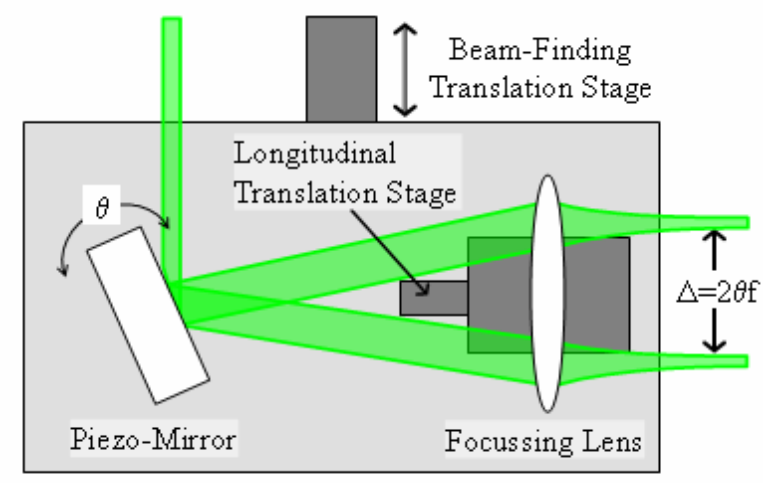

Figure 2. Schematic representation of the scanning unit (similarly designed for both scan axes). Rotating the piezo-mirror by an angle $\theta$ will deflect the beam by twice as much. This corresponds to a shift of the waist position $\Delta=2 \theta$ f (scan range). The $\mathrm{x}-\mathrm{y}$ motorized translation stages allow finding the collisions and positioning the waist in the best position. Furthermore, the Beam-Finding movement allows a larger range stage-scan.

\section{Post-IP}

After the IP, the divergent laser beam is recollected by a lens which reproduces a magnified image of the laser at the IP onto a CCD camera. Calibration of this imaging system showed a magnification $\mathrm{M}=8.5$. This allowed us to know with high accuracy the laser spot-size at the IP (otherwise impossible to determine) and to be able to deconvolute the measured scans.

Using the post-IP imaging we were able to measure the laser waist at the IP. It was $14 \mu \mathrm{m}$ (radius of laser intensity at $1 / \mathrm{e}^{2}$ of the intensity distribution) which corresponds to a $\sigma$ of $7 \mu \mathrm{m}$. Also, we were able to measure the actual scan range looking at the shift on the CCD.

\section{LASER}

The high power laser pulses are generated by an injection seeded Q-switched Nd:YAG laser, with a maximum repetition rate of $20 \mathrm{~Hz}$ The laser output pulses are doubled in frequency to obtain the final wavelength of $532 \mathrm{~nm}$. A summary of the laser characteristics is shown in table 1 . The peculiarity of this laser is that the photons of the generated light pulses are forced to be emitted with a wavelength within a very narrow band by a seeder single line laser injected into the cavity at a specific time after the active medium is pumped. This forces the first emitted photons to be at the seeder wavelength and inhibits the existence of other laser modes, otherwise allowed by the laser cavity. The advantage of having a single line Q-switched laser is the pulse is smooth and the mode-beating generated by many different modes within the laser pulse, which gives rise to a modulation of the longitudinal profile (in the scale of $100 \mathrm{~s}$ of ps), is avoided. This enables the laserwire to use a cost effective laser solution, without any complicated and expensive mode locking. The smooth temporal distribution of laser light means that less data must be averaged to get a LW profile.

Table 1: Summary of laser parameters

\begin{tabular}{|l|c|}
\hline Laser Parameter & Value and Units \\
\hline Wavelength & $532 \mathrm{~nm}$ \\
\hline $\mathrm{M}^{2}$ & $1.4 \pm 0.1$ \\
\hline Pulse duration & $(5 \pm 1) \mathrm{ns}$ \\
\hline Pulse to pulse jitter & $(2 \pm 1) \mathrm{ns}$ \\
\hline Pulse energy & $(60 \pm 5) \mathrm{mJ}$ \\
\hline Pulse peak power & $(12 \pm 1) \mathrm{MW}$ \\
\hline
\end{tabular}

\section{DETECTOR}

The Compton photon detector is downstream from the IP, after a bending magnet which deflects the charged particles and separates them from the photons to be measured. The neutral beam leaves the beam pipe vacuum system via a thin window, after which the detector is placed.

The detector is composed of a $3 \times 3$ matrix of lead tungstate $\left(\mathrm{PbWO}_{4}\right)$ crystals, fixed to a matching face photomultiplier. Each crystal has dimensions of $18 \times 18$ x150 mm and an energy resolution of approximately 5.4\% measuring electrons at $6 \mathrm{GeV}$ [5]. Measuring the same electrons, the total system had an energy resolution of approximately $10 \%$. Simulations show that Comptonscattered photons (300 MeV) deposit their energy with $95 \%$ efficiency on the $3 \times 3$ crystal matrix. 


\section{DATA-TAKING}

The scans can be performed in two different ways: 1) Moving the piezo-driven mirror in order to deflect the laser beam at the lens input. The obtained shift will be Fx2 $\theta$, being $F$ the lens focal length and $\theta$ the mirror deflection angle. (ramp scan).

2) Keeping the piezo-mirror position fixed and moving the whole translation stage step by step (stage scan).

In the 1st case the maximum applied voltage of $100 \mathrm{~V}$ to the piezo-mirror defines a maximum scan range of $1.3 \mathrm{~mm}(130 \mu \mathrm{m} / \mathrm{V})$. As shown in the next paragraph, this scan range was enough to scan across the vertical profile for which the beam size was found to be around $50 \mu \mathrm{m}$. For the horizontal instead, the measured beam size was around $400 \mu \mathrm{m}$ so a simple ramp scan would not be able to include the whole electron beam. In this latter case, a stage scan was necessary for measuring the beam width.

\section{EXPERIMENTAL RESULTS}

In fig. 3 are plotted two scans taken with the VP (fig. 3a) and the HP (fig. 3b) within a few minutes from each other. The beam energy was $6 \mathrm{GeV}$ and the bunch current $0.5 \mathrm{~mA}$. The calorimeter is read out via a peak detection circuit and the maximum of the calorimeter response is digitized using a 16 bit ADC.

These points are taken in groups and averaged to obtain the laser position and the average signal with the corresponding errors. The plot shown in fig. 3a has 29 steps and each point correspond to an average of 34 laser shots for a total of 1015 Compton events. Given that the laser repetition rate is $20 \mathrm{~Hz}$, the total scan time is approximately 50 sec.

For the horizontal beam size profiler, data were taken by moving the translation stage over $4 \mathrm{~mm}$ in steps ( $100 \mu \mathrm{m}$ for the data plotted in fig.3b), sending some number of laser pulses (20 per step in the depicted case) and averaging the calorimeter readout to give a mean value and an error. Total scan time in this case was approximately $2.5 \mathrm{~min}$. Both the vertical and horizontal beam profile measurements are fitted to the sum of Gaussian distribution and a constant. Given the laser beam size is significantly smaller than the electron beam the $\sigma$ from the fitted Gaussians can be interpreted as the electron beam size. The measured size of the electron beam vertically was $39.5 \mu \mathrm{m}$ and horizontally was $377 \mu \mathrm{m}$ for the data sample presented, giving an aspect ratio of approximately 9.5 .

\section{CONCLUSION}

In conclusion, we demonstrated that it is possible to obtain a 2D profile measurement of the beam size within few minutes from each other. There was a difference of 1 order of magnitude between the horizontal and the vertical profile which required using two different ways of measurement. Where the beam is small enough to be accommodated in the scan range, a faster measurement
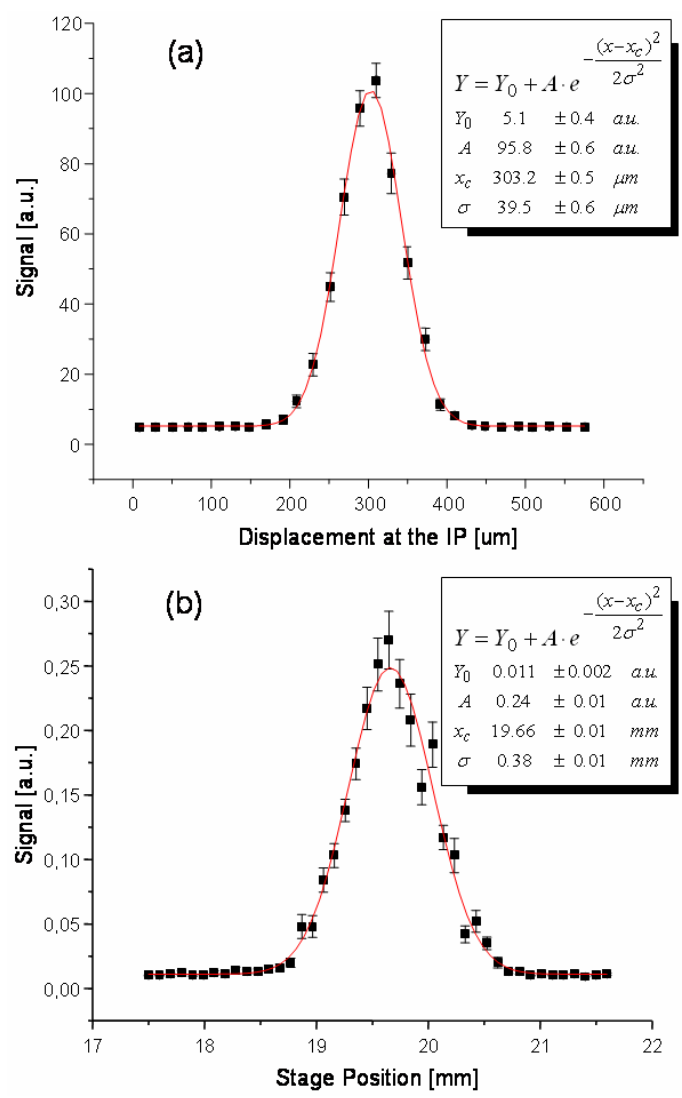

Figure 3. (a) Vertical profile measurement obtained scanning light across the electron bunch by moving the scanning mirror (scan time $50 \mathrm{sec}$ ). (b) Horizontal profile obtained moving the laser by means of the translation stage (scan time $150 \mathrm{sec}$ ).

(less than 1 minute long) can be performed with a reasonably small error. In the other case, the other type of scan had to be performed requiring longer time but still reasonably short.

In the near future, a similar LW is planned to be installed as a permanent beam profiler at PETRA III. Furthermore, a high repletion rate LW (matching PETRAIII revolution time) is being developed and will be tested at PETRA III for R\&D towards intra-train profiling at the ILC [3].

\section{REFERENCES}

[1] L. Deacon et al, "ATF extraction line laser-wire system”, PAC07 invited oral contrib.: THOAC01

[2] A. Bosco et al, Proc. EPAC06

[3] A. Bosco et al, PAC07-Poster: MOPAN110

[4] J. Carter et al., "Beam Profile Measurements and Simulations of the PETRA Laser-Wire”, Proc PAC05 (2005).

[5] F. Poirier. "Beam Diagnostic Laser-wire and Fast Luminosity Spectrum Measurement at the Linear Collider”, PhD thesis University of London, 2005. P56-90. 